\title{
Speech Motor Development in Childhood Apraxia of Speech: Generating Testable Hypotheses by Neurocomputational Modeling
}

\author{
H. Terband ${ }^{\mathrm{a}, \mathrm{b}} \quad$ B. Maassen ${ }^{\mathrm{a}, \mathrm{b}}$ \\ ${ }^{a}$ Medical Psychology/Pediatric Neurology Centre/ENT, Radboud University Nijmegen Medical Centre, \\ Nijmegen, and ${ }^{\mathrm{b} C e n t r e ~ f o r ~ L a n g u a g e ~ a n d ~ C o g n i t i o n, ~ a n d ~ U n i v e r s i t y ~ M e d i c a l ~ C e n t r e, ~ U n i v e r s i t y ~ o f ~ G r o n i n g e n, ~}$ \\ Groningen, The Netherlands
}

\section{Key Words}

Childhood apraxia of speech - Speech motor development • Computational neural modeling $\cdot$ Neuromotor deficits

\begin{abstract}
Childhood apraxia of speech (CAS) is a highly controversial clinical entity, with respect to both clinical signs and underlying neuromotor deficit. In the current paper, we advocate a modeling approach in which a computational neural model of speech acquisition and production is utilized in order to find the neuromotor deficits that underlie the diversity of phonological and speech-motor symptoms of CAS. Based on existing approaches and behavioral data, we first generated specific hypotheses about the underlying deficits. These hypotheses were then tested in a series of computer simulations, and the resulting speech patterns were compared to the available behavioral data. Finally, the model was used to derive further predictions that can be tested empirically in behavioral experiments and possible new angles for clinical intervention.

Copyright $\odot 2010$ S. Karger AG, Basel
\end{abstract}

\section{Introduction}

Speaking can be considered one of the most complex skills that humans perform. Although infants possess an inborn capacity to learn verbal communication, it takes years to acquire the pragmatic, linguistic, and perceptual-motor knowledge and skills required for the production and perception of speech. In the development of speech, 2 main stages can be distinguished. The first is a perceptual-motor stage, during which the infant acquires control over the articulo-motoric system by establishing an internal model $[1,2]$, i.e. a systemic mapping between the movements and their auditory and somatosensory consequences. This stage is usually characterized as the babbling stage $[3,4]$. The second, phonological stage comprises the acquisition of the phonological system of the language, for perception as well as production. In this stage, the perceptual-motor skills acquired thus far are further shaped and exploited to produce the first meaningful utterances, resulting in a set of phonemic mappings between articulatory movements and specific goals (which could be either auditory [5] or gestural [6] in nature). A common example of phonemic shaping is the acquisition of the /r/ versus /1/ distinction for English, and the loss of this phonemic distinction after babbled production of both sounds in the Japanese phonemic system.

All children struggle with the acquisition of systemic and phonemic mappings at a certain point, but most children come through successfully. Children with childhood apraxia of speech (CAS), however, keep struggling with the planning and execution of motor commands at the stage where they want to produce the phonemes of meaningful speech. As a clinical entity, CAS is highly controversial both with respect to clinical signs and with

\section{KARGER}

Fax +41613061234

E-Mail karger@karger.ch

www.karger.com
(C) 2010 S. Karger AG, Basel

$1021-7762 / 10 / 0623-0134 \$ 26.00 / 0$

Accessible online at:

www.karger.com/fpl
Hayo Terband

Medical Psychology/Pediatric Neurology Centre/ENT

Radboud University Nijmegen Medical Centre, Internal code 840, PO Box 9101

NL-6500 HB Nijmegen (The Netherlands)

Tel. +31 24361 3754, E-Mail h.terband@mps.umcn.nl 
respect to underlying neuromotor deficit. In the technical report by the Ad Hoc Committee on Apraxia of Speech in Children of the American Speech-Language-Hearing Association, CAS is defined as 'a neurological childhood (pediatric) speech sound disorder in which the precision and consistency of movements underlying speech are impaired in the absence of neuromuscular deficits' [7]. Despite numerous attempts, thus far studies have not yielded reliable (i.e. sensitive and specific) diagnostic markers that differentiate CAS from other types of childhood speech sound disorders (SSD). For this reason, CAS has been critically termed 'a disorder in search of a population' [8].

One of the main difficulties in the diagnosis of developmental speech disorders, and the differentiation between involvement of motor planning and phonological processes, is the evolving nature of clinical symptoms. For example, the underlying deficit in CAS is hypothesized to reside in sensory-motor capacities, but symptoms vary dramatically across the various developmental episodes of the child: starting with no or little babbling and subsequently less variegated babbling during the first year of life, slow expansion of vocabulary during the second, and producing many phonemic substitution errors in kindergarten [9]. There is large overlap in symptomatology with children with other SSD, who may also show little babbling and slow expansion of vocabulary, but for different reasons. Current clinical instruments (such as articulation tests) provide insufficient information with respect to the underlying deficit to make a differential diagnosis between SSD at a particular point in development. Differential diagnosis requires information on the developmental history of the child, combined with specific speech motor tasks and fine-grained acoustic and/ or kinematic measurements. An extensive review of the literature suggests that the clinical characteristics in CAS cannot be confined to one particular psycholinguistic stage or one particular level of speech motor control [10]. From a developmental perspective, a specific underlying speech motor impairment may affect the development of higher phonological and linguistic processing levels and vice versa, speech production problems that have a phonological origin may spread to lower (motor processing) levels $[9,10]$. This developmental interaction between higher and lower levels of processing constitutes the fundamental problem in isolating CAS from other developmental SSD. As a result, 'true' cases of CAS may not exist, but rather CAS characteristics are likely to be present to some extent in each case of developmental speech sound disorder [10]. In other words, at the symptom level, the characterization of CAS within the larger diagnosis of SSD is rather one of degree of involvement.

Instead of focusing on the clinical symptomatology of CAS, in the current paper we advocate a modeling approach, in which the concept of 'a disorder in search of a population' is not an impasse or an endpoint, but actually the starting point. Our aim is to find the neuromotor deficits that underlie the diversity of phonological and speech-motor symptoms of CAS by using computational modeling. For this, we chose the Directions Into Velocities of Articulators model (DIVA) [11, 12]. DIVA is one of the most advanced models of speech motor acquisition at the moment, and has been shown to be able to account for a variety of phonetic phenomena, such as motor equivalence, coarticulation and rate effects $[13,14]$. Furthermore, the model is linked to an articulatory speech synthesizer, which enables us to run computer simulations and generate articulatory and acoustic data that can be compared to behavioral data. Our modeling approach comprises 3 steps that form a cycle that can be repeated. First, we generate hypotheses about the underlying deficit based on existing ideas and behavioral data. Next, this deficit is implemented in the DIVA model and the resulting speech patterns are compared to the available behavioral data. Finally, the model is used to derive further predictions that can be tested empirically in behavioral experiments by comparing the model's output to experimental speech data, e.g. after manipulation of feedback, or distortion by a bite block.

\section{DIVA Modeling of CAS}

The DIVA model consists of a neural network controller detailing feedforward and feedback control loops that are involved in early speech development and mature speech production. The model strives to be biologically plausible, and its components have been associated with regions of the cerebral cortex and cerebellum. The first stage of learning in the model resembles the babbling stage. Semi-random articulatory movements are used to train a forward model, comprising the relations between motor commands and their auditory and somatosensory consequences. Once the forward model is acquired, the system 'knows' what movements to make in order to produce an auditory target, and in the next developmental phase (resembling the phonological stage) this information is used for the purpose of imitation. Motor commands for new speech sounds are acquired by the model iteratively attempting to produce a pre-specified auditory 
target region that is stored in the model's speech sound map. Based on the information provided by the auditory feedback control subsystem, the feedforward command is updated with each attempt, and thus becoming more accurate. A detailed description of the model and its computational implementation can be found elsewhere [11, 12].

\section{CAS in DIVA}

In psycholinguistic terms, CAS has been interpreted at various levels: lexical representation, phonological planning, assembly of a phonetic program and motor implementation [10]. However, the core deficit of CAS is thought to reside at the phonetic programming level [15, 16], which - in terms of psychomotor function - can be summarized as an inability to transform an abstract phonological code into motor speech commands [17]. As to what causes this deficit in generating motor speech commands, research has not yielded clear results thus far. In the DIVA model, the motor (feedforward) commands for different speech sounds are stored in feedforward projections which specify articulatory trajectories that will produce the desired auditory target. If the realized auditory (feedback) signal does not correspond to the desired auditory target, the feedforward control subsystem will update the feedforward command in order to be more accurate for the next production (also, an online corrective motor command will be calculated by the feedback control subsystem during the production; this is discussed in more detail in the next section). In other words, the coupling between the auditory target (phonetic plan) and its motor command (motor program) is established by the feedforward control subsystem. A problem in generating motor speech commands can thus be simulated in the DIVA model by poor feedforward control.

The question that arises subsequently is what underlying neurological mechanisms could cause the poor feedforward control. In this respect, we have presented 2 hypotheses [18]. The first hypothesis seeks the core deficit of CAS in a reduced or degraded oral sensitivity. Children with CAS are often observed to have a lowered oral sensitivity of the tongue and palate $[19,20]$. In DIVA, such a lack of somatosensory information would have different, but cumulative, effects in successive stages of speech development. First, during the babbling stage, uncertainty in the somatosensory state would cause weak or underspecified somatosensory-to-motor projections (synaptic projections from somatosensory error mappings to the articulator velocity map in motor cortex). Furthermore, a poor estimate of the somatosensory state will cause a poor estimate of the motor state, which subsequently would slow down the learning of auditory-to-motor projections (synaptic projections from auditory error mappings to the articulator velocity map in motor cortex). Subsequently, this would lead to degraded feedforward control in the imitation and performance stages. As the readout of the appropriate feedforward commands depends on knowing the current somatosensory state, a lack of somatosensory information would cause the readout of feedforward commands to be impaired, thus leading to degraded feedforward commands. Furthermore, the unstable somatosensory-to-motor projections would lead to degraded somatosensory feedback control in the performance stage.

The second hypothesis that was presented explains CAS as resulting from an increased level of neural noise. Neural noise has been widely associated with the tokento-token variability that characterizes human motor performance [21-23]. Neural noise is viewed as the primary factor limiting the possibility of simultaneously rapid and accurate movements, forming the main argument for the existence of paired forward and inverse internal models. Through the combination of forward and inverse models, the central nervous system can optimally estimate a current state of the system [24]. In the DIVA model, neural noise could affect all neural maps. During the babbling stage, uncertainty with respect to the current motor state and the auditory and somatosensory consequences of articular movements would slow down learning, causing weak or underspecified synaptic projections from both auditory-to-motor and somatosensory-to-motor space. In further stages, this would be expected to result not only in incorrect and/or imprecise feedforward commands, but also in poor performance of the auditory and somatosensory feedback control systems.

In a series of computer simulations we tested whether these 2 deficits lead to CAS-like behavior in the DIVA model [25]. Before going into these simulations in more detail, we will first discuss 2 predictions that the two hypotheses have in common: weak forward models and overreliance on feedback control.

\section{Weak Forward Models}

First, the 2 deficits have in common that, in the DIVA model, they would both lead to weak or underspecified synaptic projections from auditory and somatosensory error mappings to the articulator velocity map in motor cortex. In other words, both hypotheses predict that children with CAS suffer from impairment of the forward model. A recent behavioral study that was conducted in 
our laboratory yielded a number of findings that confirm this prediction [26]. Using electromagnetic midsagittal articulography (Carstens Medizinelektronic $\mathrm{GmbH}$ : Ag100), the stability and composition of functional synergies for speech movements were investigated in 10 sixto nine-year-olds with developmental speech disorders (5 diagnosed with SSD and 5 with subtype CAS) and 6 age-matched normally developing children. Articulographic data were collected in a reiterated speech task comprising the words /spa:/ and /pa:s/. The study focused on movement data of fluent speech motor behavior in perceptually correct utterances. The stability of speech motor execution was assessed by the cyclic spatiotemporal variability index (cSTI) of the movement trajectories of jaw, lower lip and tongue tip. The cSTI is a measure that captures the variability of direction-specific cyclic movement patterns $[27,28]$. The composition of synergies was assessed by the amplitudes of the movement components of tongue tip and lower lip in the realization of the constrictions for respectively the /s/ and the $/ \mathrm{p} /$ and the amplitude of jaw opening during the production of /a:/.

Results showed a higher variability of tongue tip movement trajectories in children with CAS compared to normally developing controls, indicating weak or underspecified functional synergies/coordinative structures. Previous studies have shown variability to decrease with maturation and during linguistic/phonemic development [29-32]. The increased variability found for CAS in this study therefore does not necessarily reflect a core impairment, but could also reflect developmental delay. Regarding the variability in lower lip and jaw movement trajectories, results showed no statistical differences between groups. This apparent discrepancy might be explained by the oral structure of the tongue having more degrees of freedom than lower lip and jaw. As a result, lower lip and jaw functional synergies are easier to stabilize than tongue tip, which is also supported by findings that tongue tip stabilizes later in development [33]. Although no differences were found in the variability of lower lip movement trajectories, the results did show a larger contribution to oral closures of the lower lip for CAS compared to controls. Behavioral experiments have shown that infants rely largely on the jaw in the realization of oral closures. The relative contribution of the lower lip increases as the speech production system matures $[34,35]$. At the age of around 7 years, children show still a smaller relative contribution of lower lip to the closure for the $/ \mathrm{p} /$ compared to adults [33]. The larger contribution of the lower lip for CAS compared to controls can therefore be explained by the notion that larger movements are potentially stabilizing and might constitute an adaptive strategy [27]. Overall, these findings correspond to the prediction that children with CAS suffer from a disordered development of the functional synergies/ coordinative structures that underlie speech motor coordination, thus reflecting impairment of the forward model.

\section{Overreliance on Feedback Control}

Second, in the DIVA model, poor feedforward control/ degraded feedforward commands (which both hypothesized deficits set out to explain) will lead to an overreliance on the feedback control subsystem. If feedforward commands are incorrect and/or imprecise, the articulatory movements are off-target. As a result, the realized auditory and somatosensory (feedback) signals will not correspond to the desired targets, leading to error cell activation in the auditory and somatosensory error maps. Consequently, a corrective motor command will be calculated by the auditory and somatosensory feedback control subsystems. This whole process takes place online, during the production of an utterance. Thus, the introduction of errors due to poor feedforward control causes the system to rely more heavily on the sensory feedback control subsystems [18].

The prediction that the speech motor control of children with CAS is biased towards feedback control was investigated in a first simulation study in which the reliance on feedback control was systematically varied during production attempts in the acquisition of forward commands (i.e. the stage of imitation learning) [18]. The feedforward/feedback ratio ranged from $90 / 10$ to $55 / 45$ in steps of 5/5. A total number of 27 (V1CV2) utterances were used as speech sound targets, systematically varying /a/, /i/ and /u/ for the vowels and /b/, /d/, and /g/ for the consonants. The simulations were evaluated acoustically on 4 selected key phonetic characteristics of CAS, which are indicative of CAS during the stage of imitation learning: deviant coarticulation, speech sound distortion, searching articulation and increased variability. Coarticulation was measured by the absolute differences in formant frequencies across contexts. The amount of speech sound distortion was calculated by averaging the absolute differences in formant frequencies of each produced vowel relative to the frequencies of the target vowel. Searching articulation was quantified by the mean variability in formant frequencies over time, within productions of speech sounds. Finally, variability was measured by the error variances in the formant frequencies of all productions of 
the same speech sound. These measures, accompanied by formulas, have been described in detail previously [18].

Overall, the simulation results showed an increase in all 4 key symptoms as the reliance on feedback control increased, corresponding to the prediction that in CAS, the speech production system suffers an increased reliance on auditory feedback control due to incorrect and/ or imprecise feedforward commands. Furthermore, the results showed specific differences between anticipatory and carry-over coarticulation in the consonants. For high feedforward/feedback ratios, anticipatory coarticulation was found to be equal to carryover coarticulation, but with feedforward/feedback ratios lower than $80 / 20$, carryover coarticulation was stronger. Anticipatory and carryover coarticulation have not yet been compared in a single experimental design for this pathological group (and only rarely for pathological speech in general). Our simulations predict a difference between carryover and anticipatory coarticulation in the speech of children with CAS compared to normally developing controls. Furthermore, the reduction in speech rate is known to facilitate the use of auditory feedback, thus neutralizing the effect of overreliance on feedback control [36]. Therefore, it is predicted that this difference disappears when speech rate is slowed down.

\section{DIVA Simulations of Underlying Deficits}

Subsequently, we tested whether the 2 hypotheses (reduced/degraded somatosensory information and increased levels of neural noise) lead to CAS-like behavior in the DIVA model [25]. The DIVA model features a noise generator that enables Gaussian random noise to be added to the cell activations of motor, auditory and somatosensory cortices. The level of the noise can be manipulated by specifying the mean and standard deviation of the Gaussian distribution. Reduced/degraded somatosensory information was implemented as noise in the somatosensory and motor state representations (i.e. the cell activations in the model's somatosensory and motor cortices). In the case of increased levels of neural noise, we added noise to the motor, auditory and somatosensory cortices. In both cases, the noise was zero-mean noise while the standard deviation was increased systematically from 0 to $25 \%$ in $5 \%$ steps. A detailed description of the implementations can be found elsewhere [25].

The simulation series comprised 2 stages: babbling and imitation learning. First, we trained a forward model for each of the experimental conditions, which was then used correspondingly in the acquisition of feedforward commands. The methodology of the imitation learning simulations was largely the same as in the previous simulation study [18] with the only difference that each condition was repeated 5 times, allowing token-totoken variability to be measured by the error variances in the formant frequencies of repeated productions. To differentiate the effect of noise during acquisition from the effect of system noise alone, we investigated the effect that both deficits have on speech production after asymptotic learning as control conditions.

Corresponding to our predictions, the simulations of the babbling stage showed decreased forward model performance with increased intensity for both deficits, but on different parameter levels. Furthermore, the results showed the effect to be much larger for increased levels of neural noise than for reduced/degraded somatosensory information. As discussed in the previous section, a recent behavioral study has revealed differences in the stability and composition of functional synergies for speech movements for children with CAS compared to controls. These results indicate impairment of the forward model, but unfortunately they do not tell us anything about the level of impairment. Therefore, it is not possible to make a quantitative comparison between the behavioral data and the simulation results. Obtaining data that enables the quantification of specific characteristics of forward models in human speakers constitutes a challenge for further research.

With respect to imitation learning, results showed a general increase in severity of the 4 selected key symptoms of CAS - deviant coarticulation, speech sound distortion, searching articulation and increased token-totoken variability - for both deficits, but the effect was larger for reduced/degraded somatosensory information. Lacking a quantitative comparison with observations in children with CAS, in this respect the results did not differentiate directly between the hypotheses. However, the comparison between the involvement of the deficits during and after learning did yield interesting differences between the 2 deficits. For reduced/degraded somatosensory information, results indicated that the acquired motor commands are inherently unstable/deviant, whereas increased levels of neural noise did not lead to inherently deviant or unstable motor commands. For increased levels of neural noise, results implied that the increase in anticipatory coarticulation, speech sound distortion and token-to-token variability should be attributed mainly to production/execution processes. The prediction that children with CAS do not suffer inherently deviant motor commands is directly testable in auditory and articulatory perturbation experiments [25]. 


\section{What Does This Approach Learn Us about CAS?}

Utilizing the theoretical framework of DIVA, 2 hypotheses were formulated about the neurological deficit underlying CAS. These neurological deficits - in DIVA implemented as computational deficits (neural noise; weak somatosensory processing) - have consequences for the overall architecture of the speech production mechanism in children with CAS: a weak forward mapping in combination with overreliance on feedback control. By running computational simulations of the process of speech acquisition, the behavioral consequences of these underlying deficits can be tested. First, the simulation studies showed that some of the core features of CAS emerge if these computational deficits are introduced in DIVA. Second, the simulations allow us to make predictions on the effect that manipulations of speaking conditions will have on the speech motor behavior of children with CAS. Both issues are discussed in more detail further on.

\section{Issues of Specificity}

An important point for discussion is the specificity of the simulation results; i.e. would other computational deficits lead to the same speech symptoms? In this respect, it should be noted that a certain overlap is inevitable. Many examples have shown that different underlying disorders may produce similar symptoms [10, 37]. For this reason, the point has been raised that differential diagnosis of developmental speech disorders in particular, and the study of motor speech disorders in general, should focus on characterizing profiles of symptoms instead of single diagnostic markers and strict classifications [10].

The simulation studies discussed in this paper exemplify this. The simulation results do not differentiate directly between the two deficits hypothesized to underlie CAS; both lead to poorer speech output with similar characteristics. To decide between the two hypotheses, measurements of speech production under specifically manipulated circumstances are needed, such as auditory and articulatory perturbation experiments. For example, we propose an experiment in which auditory feedback is masked and in addition an articulatory perturbation is applied. The participants' task would consist of producing simple words/syllables of which vowel quality, coarticulation and variability are measured. In the first instance, we would investigate the differences between the productions with and without masking noise applied through headphones. The lack of auditory feedback control is predicted to affect speech production more if the motor commands are inherently deviant/unstable (as is the case with degraded somatosensory information), since this can no longer be compensated by (over)reliance on auditory feedback. If the speech problems stem mainly from increased levels of neural noise during motor execution processes (with stored motor commands being intact), the role of (over)reliance on auditory feedback is more likely to be instigative than compensative and hence the lack of auditory feedback is not predicted to cause deterioration of speech production. Subsequently, adding an articulatory perturbation with a bite block is predicted to amplify this difference. Under increased levels of neural noise, the speech production system should be able to compensate immediately, because a more-or-less intact motor command is able to compensate for movement perturbation. Therefore, the bite block would not add additional deterioration. Under degraded somatosensory information, however, it would. The inherently insufficient adaptation by the poor motor commands cannot be compensated for when the use of auditory feedback control is blocked by masking noise. Thus, an interaction is expected: with masking noise a further deterioration in speech output due to articulatory perturbation is expected under degraded somatosensory information, but not (or less) under increased levels of neural noise.

Back to the notion that different core deficits can have the same consequences. The theoretical framework utilized in this paper also provides insight into the relationship between core deficit, derived or consequential deficits, and adaptive strategies. Derived or consequential deficits are deficits that emerge during development because the core deficit interferes with the normal developmental trajectory. In the field of developmental neurocognitive disorders, emphasis is on heterogeneity both at the genetic-biological level as at the phenotypic-behavioral level [38]. Thus, development itself, in addition to innate starting points, creates variation and explains the emergence of individual differences in expression and comorbidities [39]. Two mechanisms are at work. Specific underlying sensorimotor impairment may spread to different processing levels ('vertically'), and may also spread to different parts of the sensorimotor system ('horizontally'). An example of vertical spread is phonological development staying behind due to deficits in auditory processing or speech motor output difficulties. An example of horizontal spread was investigated in the first simulation study. The core impairment in CAS is thought to reside in weak forward models and the DIVA model predicts that poor feedforward control leads to an overreliance on feedback control, as a derived deficit or adaptive 


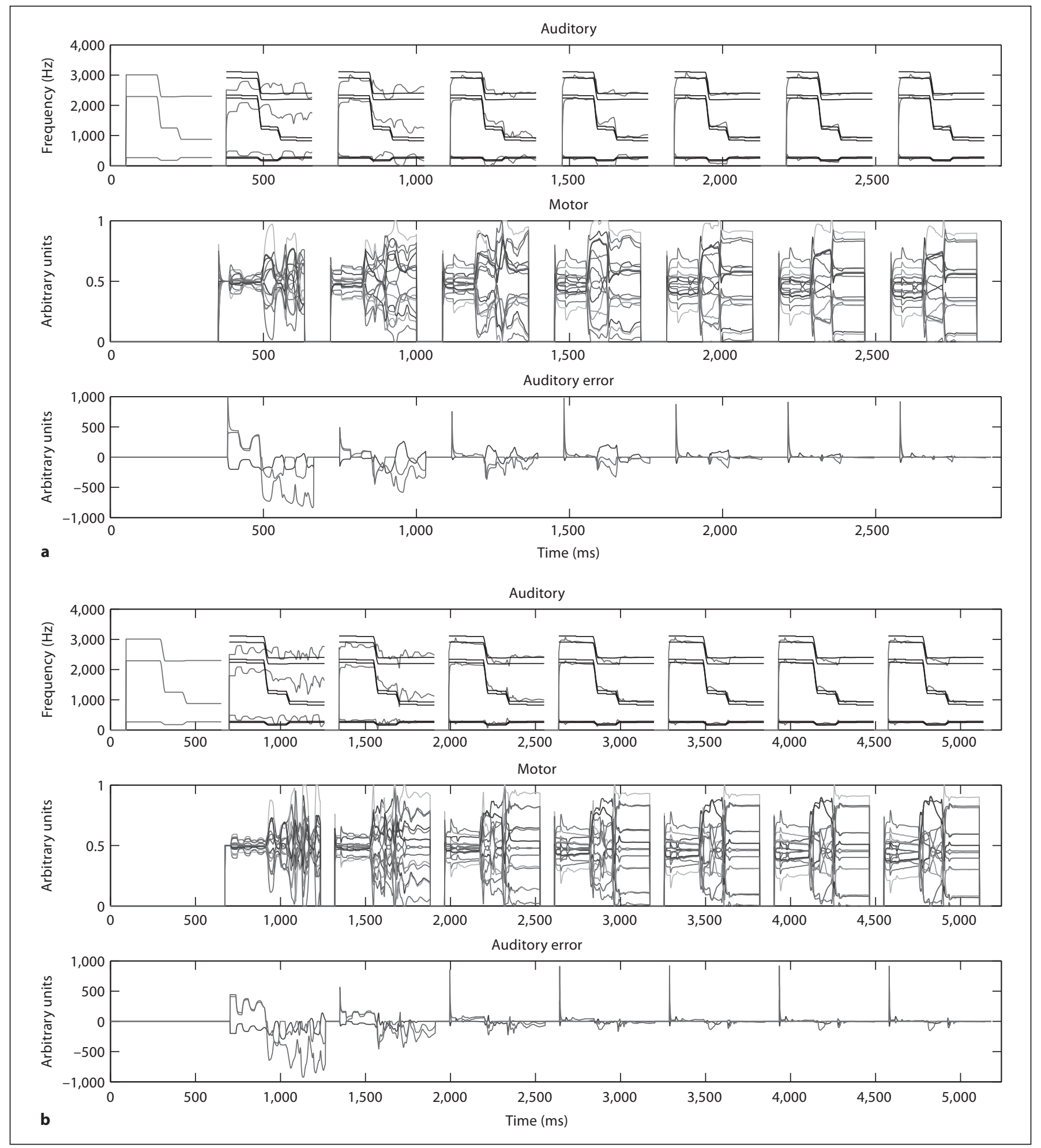

Fig. 1. The CAS-DIVA model learning the word /ibu/ at a normal/ fast (a) and at a slow articulation rate (b). The top-panel shows the auditory target (consisting of regions in F1;F2;F3-space) and the model's auditory realization. Based on the information provided by the auditory feedback control subsystem (middle-panel), the feedforward motor command (bottom-panel) is updated with each attempt, thus becoming more accurate. In the normal articulation rate condition (a), learning becomes asymptotic at approximately 7 iterations. When articulation is slowed down with a factor of 2 (b), 5 iterations are needed for learning to become asymptotic. 
strategy. The simulations showed that the isolated implementation of overreliance on feedback control leads to CAS-like behavior in the DIVA model, thus indicating that this mechanism is at work in CAS. However, overreliance on feedback control has also been proposed as the basic mechanism that causes stuttering, based on similar simulations with the DIVA model as conducted in our study $[36,40]$. In the case of stuttering, the overreliance on feedback is thought to be due to impaired readout of feedforward control and is combined with a reset function. Simulations have shown that overreliance on feedback control leads to production errors that can cause the motor system to reset and repeat the current syllable if they exceed a certain threshold [36]. This exemplifies that the same (derived) deficit may be involved in different disorders: depending on the parameter values of feedback and reset, an overreliance on feedback control can result in different phenomena, respectively groping (as in the our simulations) or stuttering.

\section{A New Angle for Clinical Intervention?}

We would like to take the parallel between CAS and stuttering one step further. In stuttering, it has frequently been reported that fluency is increased when speech rate is slowed down [41]. Correspondingly, simulations of stuttering with the DIVA model have shown that slowing down speech production reduces the size of the error and consequently the number of stutters [36]. These findings suggest that by giving the speaker ample opportunity to make use of auditory feedback (such as by slowing down articulation), otherwise disadvantageous consequences of overreliance on feedback are neutralized. Thus, slowing down articulation might constitute a new angle for clinical intervention in CAS. From motor literature it is known that practice consisting of only correctly executed trials (by taking measures to prevent errors) is more effective during particular phases of the acquisition process than practice in which erroneous productions also occur, because incorrect productions can interfere with the learning process [24]. Thus, it is predicted that in CAS slowing down articulation, and thereby facilitating the use of auditory feedback, will enhance the learning of motor commands. This mechanism is exemplified by a small computer simulation with the parameters of DIVA set to overreliance on feedback (as above), but now in combination with decreased articulation rate. The feedforward/feedback ratio was set to $75 / 25$, which is presumed to correspond to moderate CAS [18]. The simulation comprised the acquisition of the forward (motor) command for the word /ibu/ with 2 different articulation rates, a normal/fast rate and a rate twice as slow. Figure 1 presents the learning trajectories. Results show that the model needs less iterations for learning to become asymptotic when the articulation rate is slowed down, thereby confirming the predicted interaction between (over)reliance on auditory feedback and slowing down speech rate.

\section{Summary and Conclusions}

CAS has been associated with a wide variety of diagnostic descriptions and has been shown to involve different symptoms during successive stages of development. The search of the underlying deficit of CAS has not yielded clear-cut results thus far. In the current paper, we advocated a modeling approach to find the neuromotor deficits that underlie the diversity of phonological and speech-motor symptoms of CAS. Based on existing approaches and behavioral data, we first generated specific hypotheses about the underlying deficits. The computational neural model of speech acquisition and production called DIVA was then utilized to simulate these deficits, and the resulting speech patterns were compared to the available behavioral data. Finally, the model was used to derive further predictions that can be tested empirically in behavioral experiments.

Utilizing this approach, we have investigated two possible core deficits of degraded forward control in CAS: reduced/degraded somatosensory information and increased levels of neural noise. Although the DIVA simulations do not differentiate directly between the hypotheses, they do lead to directly testable predictions for auditory and articulatory perturbation experiments. Furthermore, DIVA simulations show that slowing down the articulation rate under specific conditions may be a new angle for clinical intervention. In conclusion, in the current work we have shown that simulation studies can give valuable insights in the neurological mechanisms and deficits that underlie speech disorders, and can provide fruitful guidance in the search for new clinical methods of intervention.

\section{Acknowledgments}

This study was funded by the Netherlands Organization for Scientific Research (NWO), and in part by the Fulbright Association. The authors would like to thank Frank Guenther and Jonathan Brumberg for their collaboration and support with the DIVA simulations. 


\section{References}

1 Jordan MI: Motor learning and the degrees of freedom problem; in Jeannerod M (ed): Attention and Performance XIII. Hillsdale, Erlbaum, 1990, pp 796-836.

$>2$ Wolpert DM, Ghahramani Z, Jordan MI: An internal model for sensorimotor integration. Science 1995;269:1880-1882.

3 Stark R: Stages of speech development in the first year of life; in Yeni-Komshian G, Kavanagh JF, Ferguson CA (eds): Child Phonology. New York, Academic Press, 1980, vol 1: Production, pp 113-142.

4 Oller DK: The emergence of the sounds of speech in infancy; in Yeni-Komshian G, Kavanagh JF, Ferguson CA (eds): Child Phonology. New York, Academic Press, 1980, vol 1: Production, pp 93-112.

$\checkmark 5$ Guenther FH, Hampson M, Johnson D: A theoretical investigation of reference frames for the planning of speech movements. Psychol Rev 1998;105:611-633.

$\checkmark 6$ Browman CP, Goldstein LM: Articulatory phonology: an overview. Phonetica 1992;49: 155-180.

7 American Speech-Language-Hearing Association: Childhood apraxia of speech (technical report). 2007. www.asha.org/policy.

8 Guyette T, Diedrich WM: A critical review of developmental apraxia of speech; in Lass NJ (ed): Speech and Language Advances in Basic Research and Practice. New York, Academic Press, 1981, pp 1-49.

$\checkmark 9$ Maassen B: Issues contrasting adult acquired versus developmental apraxia of speech. Semin Speech Lang 2002;23:257-266.

10 Maassen B, Nijland L, Terband H: Developmental models of childhood apraxia of speech; in Maassen B, Van Lieshout P (eds): Speech Motor Control: New Developments in Basic and Applied Research. Oxford, Oxford University Press, 2010.

-11 Guenther FH, Ghosh SS, Tourville JA: Neural modeling and imaging of the cortical interactions underlying syllable production. Brain Lang 2006;96:280-301.

12 Guenther FH: A neural network model of speech acquisition and motor equivalent speech production. Biol Cybern 1994;72:4353.

13 Guenther FH, Perkell JS: A neural model of speech production and its application to studies of the role of auditory feedback in speech; in Maassen B, Kent R, Peters HFM, van Lieshout P, Hulstijnh W (eds): Speech Motor Control in Normal and Disordered Speech. Oxford, Oxford University Press, 2004, pp 29-50.
14 Guenther FH: Speech sound acquisition, coarticulation, and rate effects in a neural network model of speech production. Psychological Rev 1995;102:594-621.

15 Schmidt R, Lee T: Motor control and learning: a behavioral emphasis. Champaign, $\mathrm{Hu}-$ man Kinetics, 1999.

16 Van der Merwe A: A theoretical framework for the characterization of pathological speech sensorimotor control; in McNeil MR (ed): Clinical Management of Sensorimotor Speech Disorders. New York, Thieme Medical Publishers, 1997, pp 1-25.

17 Nijland L: Developmental Apraxia of Speech: Deficits in Phonetic Planning and Motor Programming, 2003. http://repository.ubn. ru.nl/bitstream/2066/19265/1/19265_deveapofs.pdf.

18 Terband H, Maassen B, Guenther FH, Brumberg J: Computational neural modeling of childhood apraxia of speech (CAS). J Speech Lang Hear Res 2009;52:1595-1609.

19 Hall P, Jordan L, Robin D: Developmental Apraxia of Speech: Theory and Clinical Practice, ed 2. Austin, Pro-ed, 2007.

20 Ozanne A: Childhood apraxia of speech; in Dodd B (ed): Differential Diagnosis and Treatment of Children with Speech Disorder, ed 2. London, Whurr, 2005.

21 Harris CM, Wolpert DM: Signal-dependent noise determines motor planning. Nature 1998;394:780-784.

22 Fitts PM: The information capacity of the human motor system in controlling the amplitude of movement. J Exp Psychol 1954;47: 381-391.

23 Perkell J, Nelson W: Variability in production of the vowels /i/ and /a/. J Acoust Soc Am 1985;77:1889-1895.

24 Wolpert DM, Ghahramani Z, Flanagan JR: Perspectives and problems in motor learning. Trends Cogn Sci 2001;5:487-494.

25 Terband H, Maassen B, Guenther FH, Brumberg J: Testing hypotheses about the neurological mechanisms underlying childhood apraxia of speech (CAS). Conf Motor Speech, Savannah, 2010.

26 Terband H, Maassen B, Lieshout P, Nijland L: Stability and composition of functional synergies for speech movements in children with developmental speech disorders. Conf Motor Speech, Savannah, 2010.

27 van Lieshout PHHM, Bose A, Square PA, Steele CM: Speech motor control in fluent and dysfluent speech production of an individual with apraxia of speech and Broca's aphasia. Clin Linguist Phon 2007;21:159188.

28 van Lieshout PHHM, Rutjens CAW, Spauwen PHM: The dynamics of interlip coupling in speakers with a repaired unilateral cleft-lip history. J Speech Lang Hear Res 2002;45:5.
29 Goffman L, Smith A: Development and phonetic differentiation of speech movement patterns. J Exp Psychol Hum Percept Perform 1999;25:649-660.

30 Grigos MI: Changes in articulator movement variability during phonemic development: a longitudinal study. J Speech Lang Hear Res 2009;52:164-177.

31 Smith A, Goffman L: Stability and patterning of speech movement sequences in children and adults. J Speech Lang Hear Res 1998;41:18-30.

32 Smith A, Zelaznik HN: Development of functional synergies for speech motor coordination in childhood and adolescence. Dev Psychobiol 2004;45:22-33.

33 Terband $H$, van Brenk F, van Lieshout PHHM, Nijland L, Maassen B: Stability and composition of functional synergies for speech movements in children and adults. 10th Ann Conf Int Speech Commun Ass (Interspeech 2009), Brighton, 2009, pp 788-791.

34 Green JR, Moore CA, Higashikawa M, Steeve RW: The physiologic development of speech motor control: lip and jaw coordination. J Speech Lang Hear Res 2000;43:239-255.

35 Green JR, Moore CA, Reilly KJ: The sequential development of jaw and lip control for speech. J Speech Lang Hear Res 2002;45:6679.

36 Civier O, Tasko SM, Guenther FH: Overreliance on auditory feedback may lead to sound/syllable repetitions: simulations of stuttering and fluency-inducing conditions with a neural model of speech production. J Fluency Disord, in revision.

37 Weismer G, Kim Y: Classification and taxonomy of motor speech disorders: what are the issues?; in Maassen B, Van Lieshout P (eds): Speech Motor Control: New Developments in Basic and Applied Research. Oxford, Oxford University Press, 2010.

38 Lyytinen H: Neurocognitive developmental disorders: a real challenge for developmental neuropsychology. Dev Neuropsychol 2001; 20:459-464.

39 Karmiloff-Smith A: Development itself is the key to understanding developmental disorders. Trends Cogn Sci 1998;2:389-398.

40 Max L, Guenther F, Gracco V, Ghosh S, Wallace $\mathrm{M}$ : Unstable or insufficiently activated internal models and feedback-biased motor control as sources of dysfluency: a theoretical model of stuttering. Contemp Issues Commun Sci Disord 2004;31:105-122.

41 Van Riper C: The Nature of Stuttering. Englewood Cliffs, Prentice-Hall, 1982. 